\title{
VIOLENCIA DOMESTICA: UN MODELO GRUPAL DE INTERVENCIÓN
}

\author{
María M. Lizard'
}

I

a violencia doméstica es un problema social que afecta a un gran número de familias puertorriqueñas. También es un tema muy reseñado tanto en los medios de comunicación masiva como en la literatura profesional. El presente artículo presenta un modelo teórico-metodológico de intervención con grupos de mujeres afectadas por esta situación. El modelo a describir ha sido implantado por el Programa de Apoyo Familiar a Niños y Adultos (AFANA) auspiciado por el Instituto IGODI una institución sin fines de lucro, en la ciudad de Gurabo, Puerto Rico. El propósito primario del grupo es lograr cambios en la participante para que ésta pueda ganar control sobre su situación y preservar su integridad psíquica y física.

\section{Marco Conceptual del Modelo}

Son varios los componentes de la base conceptual de este modelo de intervención. Entre éstas se destacan la teoría de aprendizaje social (Bandura, 1977), la teoría de sistemas (Buckley, 1968), la

\footnotetext{
${ }^{1}$ Catedrática, Escuela Graduada de Trabajo Social Beatriz Lassalle, Universidad de Puerto Rico.
} 
perspectiva ecológica (Germain y Gitterman, 1996) la perspectiva de fortalezas (Saleebey, 1996) y la teoría feminista.

\section{Teoría de Aprendizaje Social}

De la teoría de aprendizaje social se incorpora la visión del aprendizaje como un proceso vicario donde el aprendiz incorpora la conducta de modelos observados a su repertorio de conductas (Bandura, 1977). Esta teoría provee una explicación de la conducta violenta. Además, explica el proceso de socialización de los niños como un aprendizaje mediante el cual se adoptan los modelos de conducta social. Parte de esta socialización conlleva la formación de género, la cual incluye la forma de percibir el mundo, la posición y función de las personas en el contexto social y lo aceptado o rechazado en función al género. La teoría de aprendizaje social también ofrece los fundamentos sobre los cuales desarrollar la metodología de intervención grupal utilizada con las sobrevivientes de violencia doméstica.

\section{Teoría de Sistemas}

Esta teoría explica la conducta humana como un proceso de interacción entre los componentes de la totalidad o unidad y entre éstos y el ambiente. La interacción de los componentes del sistema se percibe como una red causal en un tiempo y un contexto dado. Todo sistema tiene una frontera. La frontera del sistema se percibe como la delimitación del mismo y no como una barrera. La frontera permite los insumos del ambiente al sistema. A través de la frontera también el sistema devuelve al ambiente los insumos procesados.

La materia prima del sistema es la energía. Se entiende que la energía es la capacidad del sistema para la acción. Para que el sistema pueda funcionar tiene que poseer un mínimo de organización. Un sistema sin organización no puede utilizar adecuadamente su energía, teniendo esto consecuencias fatales para el sistema como un todo y para sus partes.

La premisa central de la teoría de sistemas establece que el todo es mayor que la suma de las partes. Este planteamiento destaca la importancia de la interacción en el funcionamiento del sistema. Basado en estos planteamientos el modelo propuesto reconoce que la violencia es un fenómeno multidimensional y multicausal. Debido a 
esto el profesional de la conducta que interviene con estas situaciones debe tener un acercamiento multifacético.

La teoría de sistemas ayuda a describir al ser humano como un conjunto de subsistemas. Slakieu (1996), en su modelo de intervención en crisis, desarrolló el concepto de perfil de la personalidad CASIC. Mediante esta conceptuación la personalidad se percibe como un sistema formado por cinco subsistemas a saber; cognoscitivo, afectivo, somático, interpersonal y conductual. En situaciones de crisis el sistema pierde su balance habitual y se afectan todas estas áreas.

La teoría de sistemas no sólo ayuda a explicar la violencia doméstica sino que contribuye a una visión dinámica del proceso de intervención. El trabajador social puede, mediante esta visión, intervenir con diferentes sistemas y subsistemas. Por ejemplo, puede trabajar con el individuo, el grupo, la familia , las organizaciones complejas, la comunidad y la sociedad en general.

\section{Perspectiva Ecológica}

La perspectiva ecológica plantea la conducta humana como transacciones constantes con el ambiente. Si el ambiente cumple con la función de proveer para que las personas llenen sus necesidades, el balance es funcional. Cuando existe discrepancia entre estos dos aspectos, se desarrollan problemas sociales como la violencia familiar. La necesidad de intervenir, no solamente con las personas sino con el ambiente para que éste responda mejor a las necesidades de los diversos sistemas humanos, es uno de los planteamientos que fundamenta la intervención profesional.

\section{Perspectiva de Fortalezas}

La perspectiva de fortalezas contribuye a una nueva visión de las personas servidas y del proceso de intervención. Esta perspectiva enfoca en los recursos personales del ser humano y en su capacidad para lograr el cambio deseado (Salleybee, 1994). Esta descarta la visión tradicional de intervención del modelo médico, en la cual el trabajador social es el experto que trata a las personas servidas como enfermos en un proceso de recuperación. Mediante la perspectiva de fortalezas se percibe a la persona servida como un participante de su propio proceso de transformación. Al adoptar esta 
visión el trabajador social se convierte en un apoyo facilitador mediante el cual la persona utiliza sus fortalezas y recursos para iniciar el proceso de ganar poder sobre su situación e iniciar la transformación. El modelo planteado descarta los tradicionales conceptos de cliente, paciente y terapia. A su vez incorpora los conceptos: participante, proceso transformador, sistemas de apoyo, recursos, situaciones, necesidades, intereses y facilitador.

\section{Perspectiva Feminista}

La perspectiva feminista plantea que la violencia doméstica tiene sus raíces en la estructuración social de las relaciones de poder entre los géneros. Esta perspectiva responsabiliza a la ideología patriarcal, adoptada históricamente por la sociedad, de haber legitimizado relaciones de dominación y subordinación entre el hombre y la mujer. La amenaza al control y subordinación patriarcal desencadena la conducta violenta en el agresor.

Para prevenir la violencia doméstica es necesario promover unas relaciones de poder de mayor igualdad para ambos géneros; tanto a nivel familiar como a nivel social en general. La igualdad y la justicia social son las mejores armas para combatir la violencia doméstica. Solamente ellas nos guiarán hacia un hogar con un ambiente saludable para la formación de seres humanos que puedan convivir en paz. Esto puede lograrse cuando las personas ganan conciencia sobre su situación y toman acciones afirmativas hacia el cambio. El grupo es un vehículo hacia este proceso de transformación.

\section{Principios Rectores del Modelo}

El modelo propuesto está basado en una serie de principios que describen la conceptuación de las personas servidas así como la metodología de intervención. Esta base filosófica es cónsona con las influencias teóricas del modelo. A continuación se presentan las mismas:

1. La conducta humana no es el resultado de un bagaje genético, ni de las influencias del ambiente, sino el producto de las transacciones entre ambos aspectos. El trabajador(a) social, en su intervención con la víctima de la violencia, debe documentarse, no sólo sobre las necesidades y fortalezas de las participantes sino sobre sus sistemas de apoyo formales e informales. 
2. Cada persona nace con un potencial de desarrollo que puede emerger si recibe el apoyo del ambiente.

3. Cuando existe un desbalance entre las cualidades nutridoras del ambiente y las necesidades de las personas, el funcionamiento social de éstas se verá afectado. Por ende, las situaciones de vida no se visualizan como patología o enfermedad. Esta visión elimina el estigma social tradicionalmente asociado con el rol del consumidor de servicios de trabajo social, particularmente la víctima de violencia doméstica.

4. El proceso de intervención profesional se visualiza como un intercambio de conocimientos, destrezas y actitudes en el cual las personas servidas tienen participación activa. Por lo tanto, las personas servidas se conciben, no como pacientes o clientes sino como participantes de un proceso de transformación y empoderamiento. Esto implica que desde la etapa inicial la participante aportará al proceso de intervención en la medida que le sea posible.

5. El proceso de intervención provee mayor énfasis al potencial de cambio y fortalezas de las personas servidas que a las limitaciones o problemas planteados.

6. El proceso de intervención se visualiza como uno de empoderamiento de las personas servidas para fortalecerse y lidiar con las transacciones del ambiente.

7. La participación del(a) trabajador(a) social en la formulación de la política pública es pertinente en todos los niveles de intervención.

8. Es responsabilidad del trabajador(a) social el utilizar el conocimiento adquirido en la intervención para fundamentar cambios en la política social.

9. El trabajador(a) social debe mantener una actitud científica que promueva el desarrollo de las personas servidas, redunde en un mejor servicio y contribuya al aumento del conocimiento profesional. 


\section{Objetivos de la Intervención}

La intervención estará dirigida al logro de los objetivos que se presentan a continuación:

1. Preservar la integridad psíquica y física.

2. Romper con el ciclo de la violencia.

3. Desarrollar destrezas sociales:

a) toma de decisiones

b) comunicación

c) manejo de sentimientos

d) asertividad

4. Conectar con redes de apoyo.

5. Incrementar la auto-estima.

6. Retar ideas irracionales que contribuyen a legitimizar la violencia.

7. Promover la flexibilización de los roles de género.

\section{Fases de Intervención}

\section{Divulgación de servicios y reclutamiento de candidatas}

En esta fase el grupo no está constituido como tal. Aquí el trabajador social inicia las actividades conducentes al reclutamiento de las participantes del grupo. Se utilizan diferentes métodos para identificar aquellas candidatas que podrían beneficiarse de la intervención grupal.

a. Coordinación con agencias y grupos de la comunidad - El/ la trabajador(a) social envía cartas a directores de programas de servicio y grupos comunitarios, informando sobre las posibles actividades, requisitos del programa y calendario. Este método no es efectivo si no se complementa con otras actividades tales como llamadas de seguimiento y reuniones. Algunas veces los directores de los programas prefieren delegar esta tarea de coordinación 
en otro empleado, por lo cual esto debe ser clarificado para obtener mayor respuesta del personal. En el programa Afana de Gurabo esta labor se facilita ya que existen acuerdos colaborativos entre sistemas de apoyo formales e informales de la comunidad.

b. Medios de comunicación masiva - Estos ofrecen espacios a las organizaciones sin fines de lucro de la comunidad para dar a conocer sus servicios y orientar a la comunidad. Bien utilizados, los medios de comunicación masiva tienen el potencial de ser excelentes vehículos para la prevención de problemas y el cambio social. Los comunicados de prensa, artículos periodísticos y programas educativos en radio y televisión son actividades de divulgación al alcance de los/las trabajadores sociales.

c. Reclutamiento dentro de la agencia - El programa Afana utiliza la orientación y consejería individual y familiar con las familias participantes del Programa. Estos otros servicios también proveen otro medio de reclutamiento de candidatas para la organización de grupos. Para hacer esto se prepara un formulario que permite al trabajador social recomendar la participación de personas en el grupo. Esto se hace en consulta con la participante.

d. Reclutamiento de participantes por participantes - El reclutamiento de participantes a través de otras participantes es uno de los métodos más efectivos. Este método revela el crecimiento de las participantes y es una forma de evaluar la efectividad del programa. Cuando una participante solicita los servicios voluntariamente, llega con una disposición mayor hacia los servicios. Si su acción es motivada por otra participante quiere decir que la intervención no sólo logró el empoderamiento personal sino que tuvo un impacto diseminador en la comunidad.

\section{Fase de cernimiento}

Una vez se han identificado las posibles candidatas para el grupo se procede a llenarle una petición de servicios. Con esta petición como base, se cita a la persona para llevar a cabo una entrevista de 
cernimiento. Esta entrevista tiene como propósito comenzar a establecer la relación con el/la trabajador/a social, establecer el contrato de servicios, comenzar la exploración del problema y establecer un plan preliminar de intervención.

Esta entrevista provee toda la información que permitirá al trabajador social y a la candidata prospectiva determinar si la intervención grupal es la más apropiada en ese momento dado. Un aspecto importante de esta fase es que a través de ella el/la trabajador(a) social conoce información sobre el trasfondo socio-económico y cultural de la participante. Este conocimiento le permitirá diseñar una metodología cónsona con las necesidades y características de la participante. Un ejemplo de esto es cuando la participante posee un grado limitado de estudios formales. En estas situaciones el/la líder grupal presta especial atención a éstos y adapta los ejercicios que requieran destrezas de lectura y escritura sin que las participantes se sientan incómodas. En ocasiones, la participante, por unas necesidades particulares, prefiere la intervención a nivel individual. Esta postura es respetada por la agencia.

Entre los acuerdos preliminares con la participante se garantiza la confidencialidad así como los límites de ésta, cuando esté en juego la seguridad de la participante o de terceras personas. También se establecen los requisitos de la agencia en cuanto a la relación profesional de ayuda.

Es importante que la orientación sobre los servicios se lleve a cabo en forma pausada, clara, específica y sencilla. Es fundamental que la persona se sienta cómoda y confiada en la entrevista. Se debe explicar el propósito de la misma y proveer la oportunidad para que la persona entrevistada pueda clarificar cualquier duda al respecto. En lo posible el/la trabajador(a) social debe proveer un ambiente físico y psicológico propicio al establecimiento de la relación profesional. Esto es importante para que la candidata sienta motivación para participar en el grupo.

Cabe destacar que cuando la participante llega a la agencia en el momento de una crisis activa el rol del trabajador social es más directivo ya que la persona pasa por un período de desorganización cognoscitiva, afectiva, somática, interpersonal y conductual. Este tipo de situación requiere proveer apoyo emocional, reducir los niveles de mortalidad y conectar con recursos de la comunidad. Una vez la persona se sienta más estable puede pasar a ser parte del grupo. 


\section{Fase Inicial}

En la fase inicial el grupo comienza como tal. Los propósitos de esta fase van dirigidos a facilitar la formación del grupo como sistema. En esta fase el líder del grupo asume un rol directivo para fortalecer las relaciones y las tareas a lograr. Es sumamente importante crear una atmósfera grupal agradable que promueva la confianza y la participación. Para esto, la trabajadora social hace arreglos en el ambiente físico. Se recomienda que las sillas se coloquen en forma circular para promover el que todos los miembros puedan tener contacto visual incluyendo a la líder profesional. El estar sentados en ese tipo de estructura provee la sensación psico-social de igualdad.

Otro aspecto importante es que el líder profesional conozca rápidamente los nombres de sus participantes y que facilite que ellas se conozcan entre sí. Para facilitar esta tarea se pueden llevar a cabo ejercicios grupales estructurados que promuevan el desarrollo de confianza y la interacción grupal.

La trabajadora social debe tener destrezas para manejar y promover comunicación grupal. Es importante también proveer la oportunidad para que las participantes expresen sus expectativas sobre el grupo. Para esto también existen ejercicios estructurados. Una vez se clarifican las expectativas se procede a establecer el contrato inicial del grupo. Este incluye el compromiso hacia el grupo, los días de reuniones, posibles temas y situaciones con los que el grupo trabajará y las normas grupales. Ese contrato puede utilizarse posteriormente para manejar situaciones de conflicto y se permite al grupo re-negociar el mismo de acuerdo a sus necesidades e intereses.

En esta etapa también el/la trabajador(a) social puede ofrecer una pre-prueba para medir conocimientos y actitudes de las participantes sobre la violencia doméstica. Esta medición puede repetirse en la etapa final para medir cambios en conducta y la efectividad del grupo. En cada sesión la líder grupal debe llevar a cabo una evaluación ya sea en forma verbal o escrita que mida la satisfacción de las participantes con el proceso grupal. Este tipo de evaluación se denomina formativa porque provee la oportunidad de hacer cambios durante el desarrollo del grupo. Este tipo de evaluación complementa la evaluación sumativa, la cual se lleva a cabo al final de la intervención grupal.

La etapa inicial representa la formación del grupo, la aparición de redes de relaciones y la preparación para el trabajo grupal. En 
esta etapa la acción está dirigida por el trabajador social con el fin de facilitar el proceso. Esta etapa puede tomar más de una sesión. $\mathrm{El} /$ la trabajador(a) social debe estar consciente de que el contenido o los temas a tratarse tienen que adaptarse a los procesos grupales y a la etapa de desarrollo grupal. También tiene que poseer destrezas para dirigir la discusión de un contenido, sin ignorar los procesos y dinámica grupales, así como las necesidades individuales de los miembros.

\section{Fase de Trabajo}

Una vez el grupo finaliza el proceso de entrada de sus miembros comienza la fase de trabajo. Le denominamos así pues marca el momento en que los componentes individuales del sistema unen sus insumos y trabajan hacia la formación de una personalidad grupal. Es en esta etapa donde se siente un nivel más alto de energía grupal y la misma se utiliza con un grado mayor de organización. La energía grupal es representada por la información o contenido discutido, por las ideas, valores y sentimientos compartidos. El grupo adquiere un estado de sinergia, es decir, un aumento de la energía disponible como resultado de una mayor interacción grupal.

En esta fase se observan diversos indicadores conductuales que nos permiten identificar la misma. Las participantes empiezan a hablar de «nosotras», "el grupo», «nuestras necesidades», «nuestros objetivos como grupo». Sin dejar de ser las personas que son, las participantes van sustituyendo su visión individual por una grupal. Aquí se observa también que las fronteras individuales de las participantes se van abriendo y los intercambios de insumos son mayores. El grupo despliega un nivel más alto de confianza para compartir necesidades, visiones y soluciones.

También se observa en esta etapa que las participantes han adquirido un nivel más alto de empoderamiento; de una conciencia de que al aunar esfuerzos se adquiere o despierta la capacidad de lograr la transformación creativa de su situación. Además, se incorpora la visión de que la violencia doméstica no es una situación de la cual ellas puedan ser culpadas sino un fenómeno social originado en la institucionalización de la opresión y la subordinación de las relaciones entre los géneros. Es aquí cuando las participantes pueden identificar el ciclo de la violencia doméstica, las causas y las implicaciones que ésta tiene para ellas y sus hijos. También identifican sistemas de apoyo formales e informales en su comunidad. En 
esta fase las participantes ganan conciencia del problema y empiezan a considerar las alternativas de acción que tienen. Esto incluye conocimiento sobre las leyes que las cobijan.

Es importante que la trabajadora social tenga un rol menos directivo en esta fase. Su rol debe ser de facilitadora de los procesos grupales, complementado por su rol de educadora ya que su acción va dirigida a promover cambios en conducta. La trabajadora social social debe tener destrezas para poder combinar el aprendizaje de un contenido específico, con los procesos grupales. Nunca debe anteponerse el ofrecer información, al proceso de interacción grupal. Ambas tareas deben armonizarse. La trabajadora social debe combinar los roles instrumentales o dirigidos a la tarea, con los roles expresivos, dirigidos a los procesos grupales. Esto promueve el desarrollo de liderazgo, asertividad y empoderamiento personal en las participantes. Estas destrezas podrán ser aplicadas a otras situaciones en las cuales las participantes se enfrentan a las demandas del ambiente.

\section{Fase de Terminación}

En la fase de terminación los miembros del grupo se preparan para la separación de los demás participantes y de la líder grupal. En esta fase se hace un recuento de los logros adquiridos por las participantes. Esto incluye la administración de una post-prueba para registrar los cambios en actitudes y conducta experimentados por las participantes.

Además, la trabajadora social puede incorporar ejercicios grupales estructurados para que las participantes evalúen cómo llegaron, qué lograron y qué les falta por lograr. Es de beneficio también permitir que las participantes ventilen sus sentimientos con respecto a la separación. El proceso de terminación requiere que la líder grupal haya ido preparando al grupo desde sus inicios para este momento.

El llevar a cabo ejercicios estructurados para la terminación ayuda a las participantes a lidiar con los sentimientos que ésta conlleva. Mediante estos ejercicios se promueve el que cada una de ellas exprese las ganancias obtenidas en el grupo y las aportaciones que ofreció a la experiencia grupal. Existen infinidad de ejercicios grupales estructurados para la fase de terminación. Lo importante es que los propósitos a lograrse estén claramente definidos para así poder hacer un uso diferenciado de técnicas grupales. 
Otra manera de manejar la llegada del final grupal es mediante la incorporación de actividades rituales y ceremoniales. En nuestra agencia se lleva a cabo una especie de graduación en la cual se entregan certificados y premios a las participantes. De esta manera ellas visualizan el momento como un éxito en sus vidas, como un escalón más hacia su auto-realización.

Como elemento cultural también se hace una celebración con música, comida y regalos. Esta actividad es diseñada por las participantes con el asesoramiento de la líder grupal. El llevar a cabo la misma no solamente las ayuda a manejar el proceso de terminación sino también a desarrollar destrezas para la planificación, las cuales podrán aplicar a otras situaciones. El grupo finaliza como sistema, sin embargo sirve de estímulo a la formación de vínculos entre las participantes que perduran aún después de finalizado el mismo. Las participantes preparan directorios y muchas veces continúan relacionándose en la comunidad.

\section{Técnicas de Intervención}

A través de las fases de desarrollo la trabajadora social puede aplicar una variedad de técnicas de intervención. Hay varios factores a considerar al utilizar las mismas, entre ellos los siguientes:

1. etapa de desarrollo grupal

2. objetivos de la sesión grupal

3. necesidades e intereses de los miembros

4. tiempo disponible

5. destrezas de la trabajadora social

6. recursos disponibles

Muchas de las técnicas que se utilizan en la intervención con individuos y familias pueden ser adaptadas y utilizadas en la intervención grupal. Ejemplos de estas técnicas son: ventilación, clarificación, apoyo, confrontación, reflejo de contenido, reflejo de sentimientos, parcialización, utilización de preguntas abiertas, resumen, recapitulación, "role-play", interpretación y escuchar activamente. Además de estas técnicas existen ejercicios estructurados 
para promover la confianza, interacción y comunicación en el grupo, para desarrollar destrezas sociales y para el manejo de conflictos.

\section{Conclusiones}

El modelo someramente esbozado representa un esfuerzo hacia la sistematización de la intervención profesional a nivel grupal, con mujeres sobrevivientes de violencia doméstica. El producto final de estas intervenciones ha resultado altamente efectivo. El trabajo grupal con la población servida facilita el proceso de aprendizaje y los cambios en conducta para promover la concienciación y el empoderamiento de las participantes. El/la trabajador/a social debe utilizar la intervención grupal con mayor frecuencia, reconociendo que la semilla del cambio está latente en las participantes. Dicha semilla germinará en la medida que comencemos a visualizar y a tratar a nuestras participantes como entes dinámicos y no como meras espectadoras de los procesos sociales.

\section{Bibliografía}

Astelarra, J. (1984). El feminismo como perspectiva teórica y como práctica política: Teoría feminista. Santo Domingo: Ediciones CIPAF.

Bandura, A. (1977). Social learning theory. New Jersey: Prentice Hall.

Beal, Carol R. (1994). "Theories of gender role development: Social learning”, Boys and girls: The development of gender roles. New York: McGraw Hill, Inc.

Bergen, R. (Ed.)(1998). Issues in Intimate Violence, Thousand Oaks, CA: Sage Publications.

Browne, A. \& Dutton, D. G. (1990). Escape from violence: Risks and alternatives for abused women-what do we currently know? Canada: British Columbia Institute on Family Violence.

Buckley, W. (1968). Modern System research for the behavioral scientist. Chicago: Aldine Publishers Co. 
Caro, E.; Mattei, M.; Medina, G.; Mercedes, M. (2000). Cuando a mami la maltratan. Tesis de maestría sin publicar. Universidad de Puerto Rico, Recinto de Río Piedras, Escuela Graduada de Trabajo Social.

Cwik, M. (1999). "A review of current research on domestic violence”. Internet URL:http://members.aol.com/agunah/ review.htm.

Cruz Diaz, E. \&cotros (1995). La más casera de las violencias sociales: Violencia contra la pareja. San Juan: Editorial de una Orilla a Otra Orilla.

Gelles, R. (1997). "Explaining intimate and family violence". Intimate violence in families. Thousand Oaks, CA: Sage Publications.

Germain, C. \& Gitterman, A. (1996). 2nd ed. The life model of social work practice. New York: Columbia University Press.

Golombok, S. and Fivush, R. (1994). "Social learning theory". Gender development, Cambridge: Cambridge University Press.

Saleebey, D.D. (1996). The strengths perspective in social work. (2nd. ed.). New York: Longman. 